\title{
Generating Synchronization Engines between Running Systems and Their Model-Based Views
}

\author{
Hui Song ${ }^{1}$, Yingfei Xiong ${ }^{1,2}$, Franck Chauvel ${ }^{1}$, Gang Huang ${ }^{1}$, Zhenjiang $\mathrm{Hu}^{3}$, \\ and Hong $\mathrm{Mei}^{1}$ \\ ${ }^{1}$ Key Laboratory of High Confidence Software Technologies (Ministry of Education) \\ Peking University, Beijing, China \\ \{songhui06, franck. chauvel, huanggang, meih\}@sei.pku.edu.cn \\ 2 Department of Mathematical Informatics, University of Tokyo, Tokyo, Japan \\ xiong@ipl.t.u-tokyo.ac.jp \\ 3 GRACE Center, National Institute of Informatics, Tokyo, Japan \\ hu@nii.ac.jp
}

\begin{abstract}
The key point to leverage model-based techniques on runtime system management is to ensure the correct synchronization between the running system and its model-based view. In this paper, we present a generative approach, and the supporting tool, to make systematic the development of synchronization engines between running systems and models. We require developers to specify "what kinds of elements to manage" as a MOF meta-model and "how to manipulate those elements using the system's management API" as a so-called access model. From these two specifications, our SM@RT tool automatically generates the synchronization engine to reflect the running system as a MOF-compliant model. We have applied this approach on several practical systems, including the JOnAS JEE server.
\end{abstract}

\section{Introduction}

The increasing need of continuously available systems (IT systems, e-business, or critical systems) requires to perform management activities such as configuration, evolution or corrective maintenance at runtime.

Management activities (automated or not) are build on a loop [1]: monitoring the running system, analyzing the collected data, planning the needed reconfigurations, and executing those reconfigurations. For monitoring and executing, existing platforms such as JEE [2], Fractal[3], and Android [4] provide adequate facilities through devoted APIs, such as the JMX API [5] for JEE systems. For analysis and planning, researchers proposed many generic approaches, utilizing model-based techniques like architecture styles [6,7], model checking [1], modelbased self-repair [8], or model-based artificial intelligence [9], etc.

The key-point to leverage model-based analysis and planning at runtime is to obtain a model-based view of a running system and to ensure the proper synchronization between the system and its model-based view.

However, despite their importance, such synchronization engines are still hand-crafted in a tedious and error-prone manner. Existing approaches $[7,10,8$, 11] include hand-written synchronization engines. To do so, developers have to care about how to maintain a model, how to manipulate the system through the 
management API, and how to propagate the changes between them to ensure their consistency. All these functionalities have to be considered simultaneously.

The contribution of this paper is to make systematic the development of such synchronization engines between models and running systems. Our approach reflects a simple model-driven process: For a specific system, we require the developers to specify what elements can be managed, and how to manipulate them through the management API. From these two specifications, our approach automatically generates a synchronization engine that maintains a dynamic MOF-compliant model for the running system. This model enables the standard model-based techniques (like OCL and QVT) to be used for runtime management. We implement this approach as a tool named SM@RT ${ }^{4}$, and apply it on several practical systems.

The rest of this paper is organized as follows. Section 2 illustrates the difficulty for developing a synchronization engine by hand whereas Section 3 presents an overview of our generation process. Section 4 and Section 5 explain how developers specify the system and how to generate the synchronization engine. Section 6 describes and discusses our case studies. Finally, Section 7 presents some related approaches and Section 8 concludes the paper.

\section{Motivating Example}

This section illustrates the complexity of developing a synchronization engine (SE) between a running systems and its model view.

We choose the JOnAS [12] JEE application server as a running example. A JOnAS server contains a lot of manageable elements such as EJBs, data sources (proxies to databases), etc. Each data source maintains a pool of connections to the underlying database. If the number of cached connections tends to reach the capacity of the connection pool, the database access may be delayed and the pool capacity must be enlarged. In the same manner, if the number of cached connections is always zero, the data source can be removed to release resources.

JOnAS provides a low-level interface (the JMX [5] management API) for the monitor and execution of manageable elements. But complex analysis and planning must still be performed by hand or by using external tools. Modeldriven techniques and tools can help such analysis and control tasks. Take the above management scenario as an example, the administrators could use a model visualization tool (like GMF [13]) to help better understand the system, or use OCL constraints to automatically verify the server reconfiguration.

Like other model-based technologies, GMF and OCL can be only applied on MOF-compliant models (as shown in Figure 1), which is constituted by standard model elements. But the JMX API represents the running system as a specific kind of Java objects, the Managed Beans (MBeans). The integration of modelbased techniques thus requires an $\mathrm{SE}$ which reflects the running system into a MOF-compliant model, and ensures a bidirectional consistency between the system and the model. For instance, in our JEE scenario, the SE must build a model element for each data sources on the JEE AS. When the management

\footnotetext{
4 "SM@RT" for Supporting Models at Run-Time: http://code.google.com/p/smatrt
} 
agent deletes a model element, the SE must detect this change, identify which data source this removed element stands for, and finally invoke the JMX API to remove this data source.

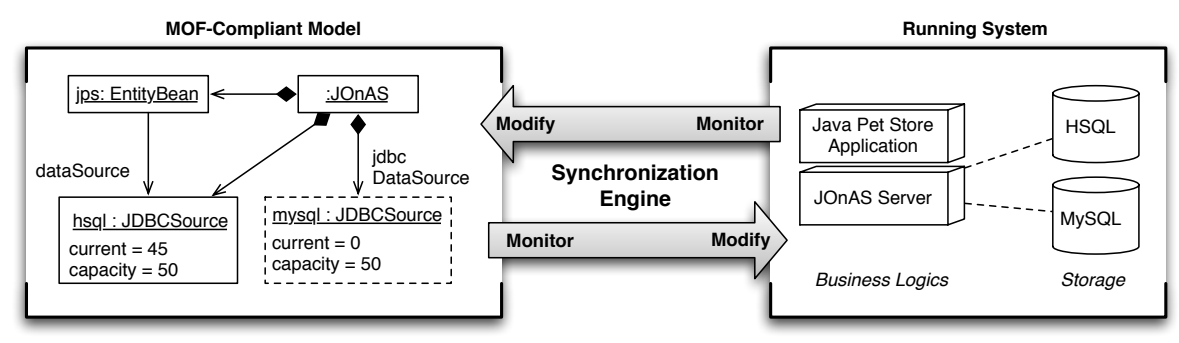

Fig. 1. A common structure of the synchronization engines

However, such an SE employs a complex mechanism and its development is therefore time consuming and error-prone. For the above scenario, the SE has to perform many functionalities: reading and writing models, monitoring and executing system changes, maintaining the mapping between model elements and system elements, handling conflicts between changes, and planning the proper subsequent changes to make the model and system consistent. In addition, SEs share many commonalities, and developing the SE from scratch is a waste of time and labor. Actually, except for monitoring and executing system changes, all the other functionalities are independent to the specific systems, and thus it is possible to achieve common solutions for them.

\section{Approach Overview}

We provide a generative approach to assist the development of synchronization engines. As shown in Figure 2, the inputs of our approach include a system metamodel specifying what kinds of elements can be managed and an Access Model specifying how to use the API to monitor and modify those manageable elements. Our SM@RT tool generates a SE which reflects automatically the running system into a MOF-compliant model that conforms to the system meta-model.

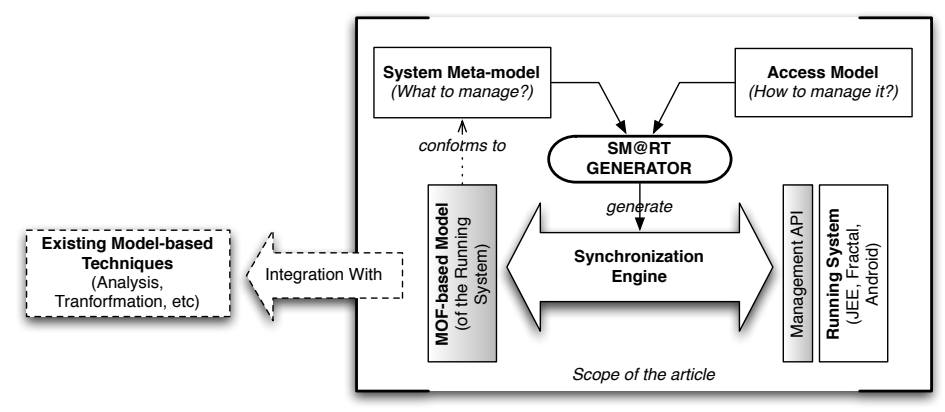

Fig. 2. Generation of Synchronization Engine: Approach Overview 
Our approach is applicable on the following premises. First, we require the target system to provide a management API: our tool does not instrument nonmanageable systems, nor extends inadequate APIs. Second, we reflect a direct model for the system (that means the model is homogeneous with the system structure: each model element stands for one system element). If an abstract model is needed, a model transformation could be used to transform this direct model into the needed forms, which is beyond the scope of this paper.

\section{Modeling Management APIs}

In order to generate an SE for a specific system, we need to know what can be managed in this system, and how to managed it. In this section, we present how to specify these two kinds of information as models.

According to Sicard et al. [8], a manageable running system is constituted of managed elements. Managed elements have local states. They could be composed by other managed elements, and they could have connections between each other. These four concepts can be described using the following four concepts in EMOF meta-model [14], i.e. Class, Attribute, Aggregation, Association, respectively. Figure 3 is an excerpt of the meta-model we defined for JOnAS.

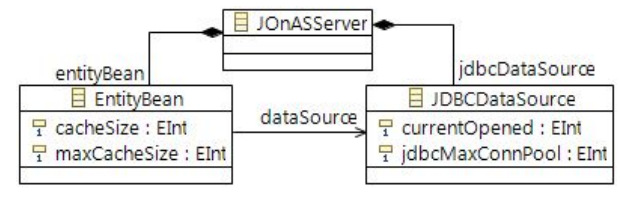

Fig. 3. The system meta-model of the JEE example

The manageable elements can be manipulated through the management API. For example, we can use getAttribute method of JMX to fetch the current opened connections of a data source. For a system, we require developers to specify how to invoke its management API to manipulate each type of elements, and we name this as an "access model". More formally, the access model is a partial function:

access : MetaElement $\times$ Manipulation $\longrightarrow$ Code

where MetaElement is the set of all the elements in the system meta-model (classes, attributes, etc.), Manipulation is the set of all types of manipulations, which are summarized in Table 1, and Code is a piece of Java code.

Figure 4 shows two sample items in the access model for JOnAS. The first item defines the common code for getting the values of int-typed attributes. We obtain an instance of an MEJB remote element (Line 4), and the logic is defined as an Auxiliary. Then we invoke the getAttribute method provided. The first parameter is the reference to the current management element. The second parameter is the property name. The second sample is for adding a new data source into a server, or "loading a data source according to a specific name" in the JEE language. We first find the model element added by management 
Table 1. All kinds of manipulations. For each kind of operation, we list its name, the types of meta elements it could applied, the parameters it required for execution, and a brief description. In the table, Property standards for attribute, aggregation and association, and the following "1" or "*" refers to the single-valued or malti-valued properties, respectively. The Auxiliarys are common operations defined by users, and can be used during the definition of code, as shown in the example.

\begin{tabular}{l|l|l|l}
\hline name & meta element & parameter & description \\
\hline Get & Property $(1)$ & - & get the value of the property \\
\hline Set & Property $(1)$ & newValue & set the property as newValue \\
\hline List & Property $\left(^{*}\right)$ & - & get a list of values of this property \\
\hline Add & Property $\left(^{*}\right)$ & toAdd & add toAdd into the value list of this property \\
\hline Remove & Property $(*)$ & toRemove & remove toRemove from the list of this property \\
\hline Lookfor & Class & condition & find an element according to condition \\
\hline Identify & Class & other & check if this element equals to other \\
\hline Auxiliary & Package & - & user-defined auxiliary operations \\
\hline
\end{tabular}

Fig. 4. Invoking JMX interface

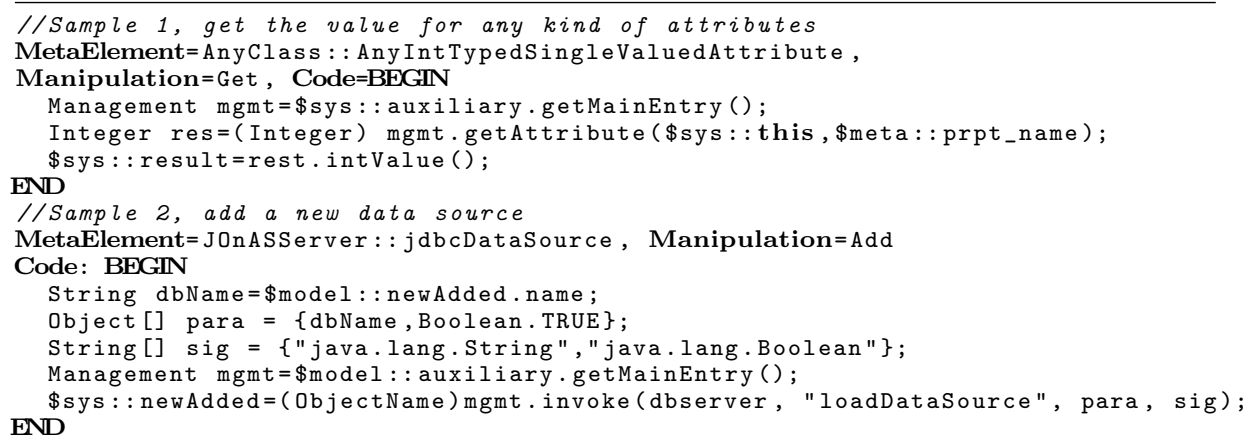

agents, and get the data source name (Line 11) from this element. Finally we use this name to invoke the loadDataSource operation (Lines 12-15).

When defining how to manipulate the systems, developers may need system information (like "what is the current system element", Line 5), system type information (like the property name, Line 5), and the inputted information by the external management agent (like the appointed name for the to-be-created data source, Line 11, such information is preserved in the corresponding model element). We defined three kinds of specification variables, the system, meta and model variables, to stand for the above three kinds of information, in order to keep developers from the details about the generation and the SE.

\section{$5 \quad$ Generating the Synchronization Engine}

This section presents the SEs we generated to maintain the causal links between model and system. We first explain how the generated SEs work, and then introduce how we generate the engines.

The first question for a synchronization mechanism is "when and where to synchronize". Since the model is the interaction point between the system and the management agent (MA), synchronization should be triggered before $M A$ read the model and after they write the model. In addition, for each reading or writing, the MA only cares about part of the model. And thus, we only 
synchronize the involved part of model with the running system. Such on-demand synchronization preserves correctness and increases performance.

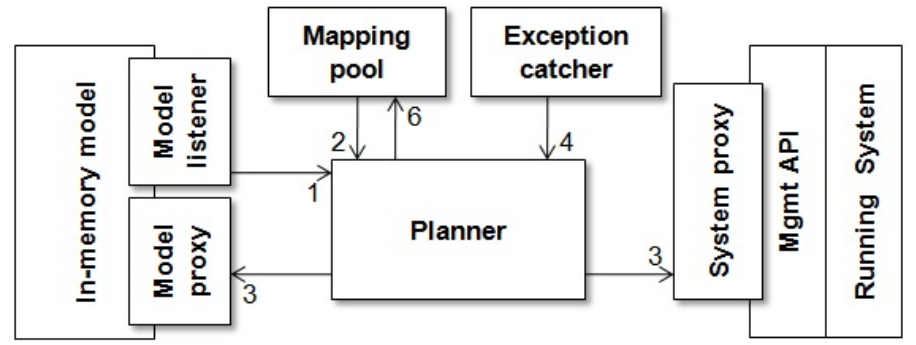

Fig. 5. Structure of the generated SE

Figure 5 shows the structure of our SE, implementing the on-demand synchronization approach we discussed before. The model we provide is in an inmemory form conforming with Ecore [13]. Each model element is represented by a Java object in the type of EObject. External management agents read or write this model by invoking the standard get or set methods on these model elements. The Model Listener listens to these model operations. For a reading operation, the listener interrupts the operation, asks the planner to do synchronize, and finally resume the original operation with the refreshed model. For a writing operation, it waits until the operation finished, and asks the planner to synchronize this modified model with the system. The Mapping pool maintains a one-to-one mapping between the model elements and the system elements, as a reference for the synchronization. The Model and System proxies are used to read the current model and system, and write the required changes (i.e. the synchronization result) back. The Exception Catcher implements a simple conflict handling strategy, i.e. when a conflict causes failures during the model or system manipulation, it catches the thrown exceptions and warns the management agent. Based on these auxiliary parts, the central planner execute a set of synchronization strategies:

$$
\text { SynchStrategy : ModOp } \times \text { MOFElem } \rightarrow(\text { ModOp } \bigcup S y s O p \bigcup M a p O p) *
$$

Each strategy defines that when a specific kind of model operations (get, set, etc.) happened on a specific part of the model (model elements, single-valued attributes, etc.), the engine will execute a sequence of operations. These operations manipulate the model, the system, and the mapping pool, in order to make them consistent.

Due to the space limitation, we do not explain each strategy, but use the following sample to illustrate how they work. For the JOnAS sample, in the beginning, the model contains only one element standing for the JOnAS server. The management agent invokes get method on this root element to see its data sources. The model listener interrupts this get operation, and informs the planner. Follow the synchronization strategy for get operations on multi-valued aggregations, the planner performs the following operations: It first checks the mapping pool to see that root stands for the JOnAS server, and then invoke 
list on this server (See Table 1), which returns a set of ObjectNames pointing to the current data sources. The planner then invokes the create operation on the model proxy to create a new model element for each of these data sources, and refresh the mapping pool for these new model elements. Finally, the original get operation continues, and returns a set of newly created model elements.

Our SM@RT tool automatically generates the above SEs. The tool has two parts, a common library and a code generation engine. The common library implements mapping pool, the exception catcher, and the planner, with the synchronization strategies hard-coded inside. The code generation engine is an extension of the Eclipse Modeling Framework (EMF), and it generates the model listener, model proxy, and system proxy specific to the target system. Specifically, it generates a Java class for each of the MOF classes in the system meta-model, implementing the EObject interface defined by Ecore. Then it overrides the model processing methods in EObject, inserting the logic for listening operations and launching the synchronization planner. Finally, it wraps the pieces of API invocation code in the access model into a set of system manipulation methods, which constitutes the system proxy.

\section{Case Studies}

We applied our SM@RT tool to generate SEs for several practical systems, and performed several runtime management scenarios on these models, utilizing existing MOF-based model-driven techniques.

\subsection{Reflecting JOnAS JEE systems}

Our first case study is the full version of the running example we used before. We reflect all the 21 kinds of JEE manageable elements (including applications, EJBs, data sources, transaction services, etc.) as a MOF-compliant model, and visual it to provide a graphical management tool for JOnAS administrators.

We first define the system meta-model and the access model for JOnAS as explained in the previous sections. The resulting system meta-model contains 26 classes, 249 attributes, 21 aggregations and 9 associations. The resulting access model defines 28 pieces of code like the sample in Figure 4.

From the system meta-model and the access model, the SM@RT tool automatically generates the SE for JOnAS as a Java library. We connected this library with GMF to visualize the reflected model (just in the same way as visualizing any common Ecore models), as shown in Figure 6.

In this snapshot, the rectangles stand for the JOnAS manageable elements and the lines stand for the association between these elements. From this diagram, we see that there are two applications running on the pku server, which runs on one JVM, and contains several resources, including a data source named HSQL1. We select the data source, and the property view on the right side shows its attribute values. All the elements, associations and attributes depict the current system state. That means if we select this model element again (that causes GMF to refresh the attributes), some attribute values may change, and if we select the canvas (that causes GMF to refresh the root element), some elements 


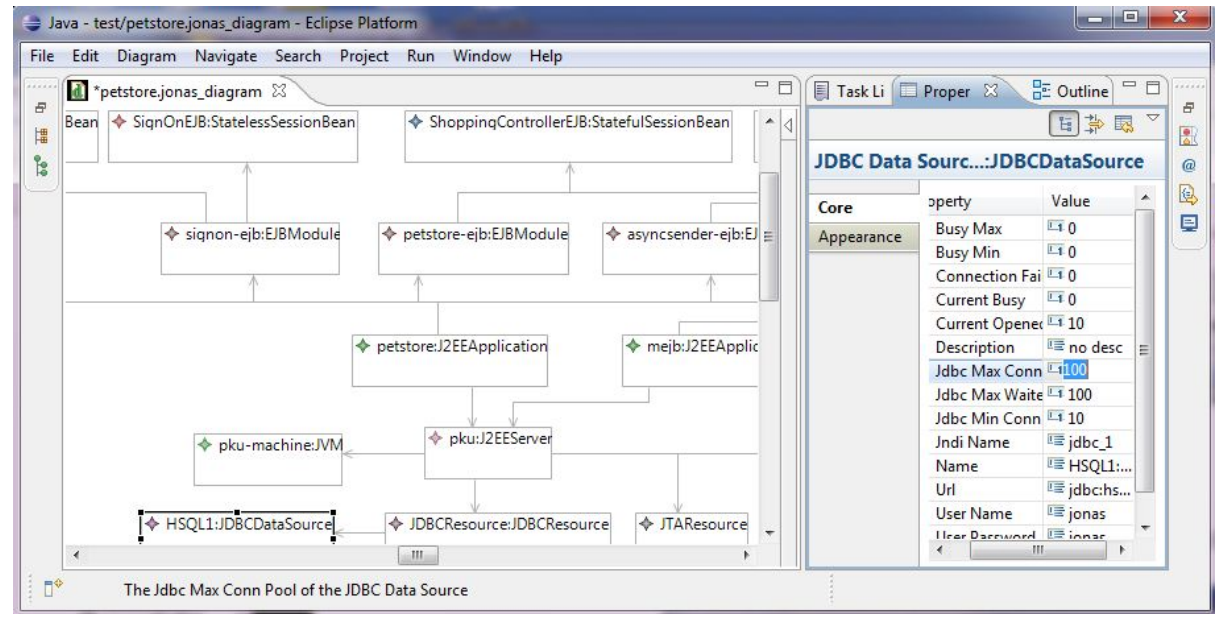

Fig. 6. A snapshot of the visualized model of JOnAS

may disappear and new elements may appear. We can also directly use this diagram to change the system. For example, if we increase the JDBCMaxConnPool from 100 to 200, the underlying pool will be enlarged consequently. If we create a new model element in the type of J2EE Application, and set its fileName attribute as the address of an EAR file, the synchronization engine deploys this EAR file into the system, and some new model elements will appear in the diagram, standing for the modules and EJBs inside this newly-added application.

\subsection{Other case studies}

Table 2. Summary of experiments

\begin{tabular}{|l|c|c|cc|c|c||l|}
\hline target system & API & $\begin{array}{c}\text { meta-model } \\
(\text { elements })\end{array}$ & $\begin{array}{c}\text { access model } \\
(\text { items })\end{array}$ & $\begin{array}{c}\text { gener }) \\
(\text { LOC })\end{array}$ & $\begin{array}{c}\text { contrast } \\
(\text { LOC })\end{array}$ & techs \\
\hline JOnAS & JMX & 305 & 28 & 310 & 18263 & 5294 & GMF \\
\hline Java classes & BCEL & 29 & 13 & 124 & 10518 & 3108 & UML2 \\
\hline Eclipse GUI & SWT & 31 & 23 & 178 & 11290 & - & EMF \\
\hline Android & Android & 29 & 9 & 67 & 8732 & - & OCL \\
\hline
\end{tabular}

Table 2 summarizes all the case studies we have undertaken. For each case, we give the target system and its management API, the numbers of elements in the system meta-model, the items in the access model and the total lines of code in these items. After that, we list the sizes of the generated synchronization engines. For the first two cases, we also list the size of the hand-written synchronization engines for comparison. Finally, we list the model-driven techniques we applied upon the generated SEs. The second case is a reproduction of the Jar2UML tool ${ }^{5}$, which reflects the class structure in a Jar file as a (read-only) UML model. The third case supports dynamic configuration of an Eclipse window, like changing a button's caption or a label's background color. The fourth case is about using OCL rules to check the package structure of Android mobile phone systems.

\footnotetext{
${ }^{5}$ http://ssel.vub.ac.be/ssel/research/mdd/jar2uml, a use case of MoDisco [11]
} 


\subsection{Discussion}

Feasibility The case studies above illustrate the feasibility of our approach: it generates SEs for a wide range of systems, and the generated SEs enable existing model-driven techniques for runtime management.

Generation Benefits Our generation approach improves the development efficiency of SEs. Among the complex functionalities of SEs (see Section 2), we only require developers to care about the monitoring and controlling of the system. Specifically, we reduce $94.1 \%$ hand-written code for the JOnAS case (310 vs. 5294 LOC), and $98 \%$ for the Java case (62 vs. 3108 LOC).

Synchronization Performance The performance of the generated SE is acceptable. For the JOnAS case, we deploy the JOnAS server and the synchronization engine on a personal computer with $3.0 \mathrm{GHz} \mathrm{CPU}$ and 2.0GB memory. We spend 3.17 seconds in average to show the diagram shown in Figure 6, with 98 manageable elements in total, and we spend less than one second to refresh an element or change an attribute. The performance is similar to the default web-based management tool, the JOnAS Admin. For the Android case, we spend 1.7 seconds to perform the OCL adaptation rule.

\section{Related Work}

Many researchers are interested on model-based runtime management. The representative approaches include "runtime software architecture" [6,15], "models at runtime" [16], etc. Currently, these approaches focus on the problems and ideas of model-based management, and implement their ideas on specific systems and models. Alternatively, we focus on the reflection of models for different systems, and try to provide automated support.

Some researchers also focus on reflecting different systems into standard models. Sicard et al. [8] employ "wrappers" to reflect systems states into Fractal models. Researchers of MoDisco Project [11] focus on developing "discoverers" to discover MOF-compliant models from systems. The "wrappers" and "discoverers" are similar to our SEs, but our work support developers in constructing SEs from a higher level, not by directly writing code in ordinary programming language. Another difference between our work and MoDisco is that our SEs support writing the model changes back to the system.

Bencomo et al. [17] also use model-to-text generation to automate system management. But currently they generate the configuration files specific to the Gridkit platform, while we try to generate SEs for various systems.

Our synchronization mechanism is related to the approaches on model synchronization [18]. The difference is that these approaches use the same model processing interface to manipulate the two participants of synchronization, but we try to integrate ad hoc management APIs into the synchronization process.

\section{Conclusion}

To efficiently leverage the use of model-based techniques at runtime, it is necessary to have a model-based view of the running system. In this paper, we 
report our initial attempt towards the automated generation of synchronization engines that reflect running systems into model-based views. We require developer to specify "what to manage on the system" as a MOF meta-model, and specific "how to use the related API to do so" as an access model. From these specifications, we automatically generate the synchronization engine that reflects the system as a direct MOF compliant model. We have successfully applied our approach on several practical systems, and enabled several typical model-based techniques at runtime. As future work, we plan to give more support for developers to specify the running systems and their APIs. We also plan to perform further analysis such as model checking to ensure a deeper correctness and completeness of the generated causal link.

\section{References}

1. Kramer, J., Magee, J.: Self-Managed Systems: an Architectural Challenge. In: Future of Software Engineering (FOSE) in ICSE. (2007) 259-268

2. Shannon, B.: Java Platform, Enterprise Edition 5, Specifications (April 2006)

3. Bruneton, E., Coupaye, T., Leclercq, M., Quema, V., Stefani, J.: The Fractal Component Model and its Support in Java. Software Practice and Experience 36(11-12) (2006) 1257-1284

4. DiMarzio, J.: Android: A Programmers Guide. McGraw-Hill Osborne Media (2008)

5. Hanson, J.: Pro JMX: Java Management Extensions. (2004)

6. Oreizy, P., Medvidovic, N., Taylor, R.N.: Architecture-based runtime software evolution. In: ICSE. (1998) 177-186

7. Garlan, D., Cheng, S., Huang, A., Schmerl, B.R., Steenkiste, P.: Rainbow: Architecture-based self-adaptation with reusable infrastructure. Computer 37(10) (2004) 46-54

8. Sicard, S., Boyer, F., De Palma, N.: Using components for architecture-based management: the self-repair case. In: ICSE '08: Proceedings of the 30th international conference on Software engineering, New York, NY, USA, ACM (2008) 101-110

9. Chauvel, F., Barais, O., Borne, I., Jézéquel, J.M.: Composition of qualitative adaptation policies. In: Automated Software Engineering Conference (ASE 2008). (2008) 455-458 Short paper.

10. Batista, T., Joolia, A., Coulson, G.: Managing Dynamic Reconfiguration in Component-Based Systems. In: Software Architecture: 2nd European Workshop, EWSA 2005, Pisa, Italy, June 13-14, 2005: Proceedings, Springer (2005)

11. MoDisco Project http://www.eclipse.org/gmt/modisco/

12. JOnAS Project. Java Open Application Server http://jonas.objectweb.org

13. Budinsky, F., Brodsky, S., Merks, E.: Eclipse Modeling Framework, project address: http://www.eclipse.org/modeling/emf

14. Catalog of OMG Modeling and Metadata Specifications http://www.omg.org/ technology/documents/modeling_spec_catalog.htm

15. Huang, G., Mei, H., Yang, F.: Runtime recovery and manipulation of software architecture of component-based systems. Auto. Soft. Eng. 13(2) (2006) 257-281

16. France, R., Rumpe, B.: Model-driven development of complex software: A research roadmap. In: Future of Software Engineering (FOSE) in ICSE '07. (2007) 37-54

17. Bencomo, N., Grace, P., Flores, C., Hughes, D., Blair, G.: Genie: Supporting the model driven development of reflective, component-based adaptive systems. In: ICSE. (2008) 811-814

18. Giese, H., Wagner, R.: Incremental model synchronization with triple graph grammars. In: MoDELS. (2006) 543-557 